ences to reviews are intermingled among those to research articles, making them hard to distinguish.

This book should certainly be considered for the institutional and private libraries of all endocrinologists. A large amount of clinical information makes it potentially useful in medical school courses. Whether or not it is suitable for use as a textbook depends on whether the course is long (e.g., two quarters or semesters), graduate, or specialized (e.g., dealing with the mechanisms of hormone action).

RONALD R. NOVALES Department of Neurobiology and Physiology

Northwestern University Evanston, IL 60201

\section{MAMMALIAN FUNCTION}

Mammalian Physiology. J. Homer Ferguson. Scott, Foresman, \& Co., Columbus, $\mathrm{OH}, 1985.517$ pp., illus. $\$ 30.05$ (cloth).

In the preface of this text, the author states that he has "attempted to introduce the student to a wide variety of physiological adaptations that enable mammals to inhabit the many habitats available to them" and that the book is designed for use in an organ-systems physiology course not focused on human or medical physiology. The book is intended to prepare the zoology or biology student for advanced courses in physiology and to provide a physiological foundation for students in applied zoology (e.g., wildlife management). Has the author achieved these goals? The answer is "sometimes."

The organ-system approach emphasizes mechanisms in the tradition of most animal physiology texts. Although this organizational emphasis provides a foundation for advanced physiology courses, the concept of physiological adaptation is lost in the lack of any environmental thrust. Modern evolutionary approaches to mammalian physiology are absent; neither evolution nor natural selection occur in the index. Comparative material does enliven the text, but is subservient to the description of mechanisms. This lack of an ecologi- cal/evolutionary approach is evident in the discussion of pregastric (ruminant) and postgastric (cecant) fermentation. The advantages of ruminant digestion are emphasized, but the greater ability of cecants to utilize low protein roughage is not discussed. Similarly, the high blood pressures of mammals are described in physical

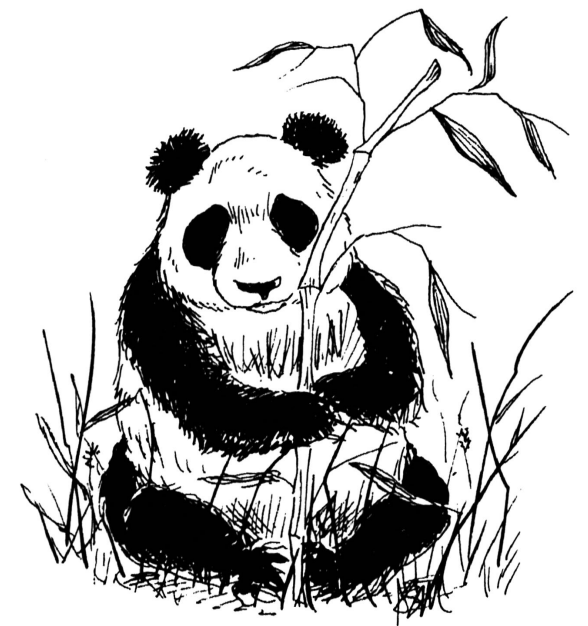

terms, but the reader never learns why mammals should have high blood pressure in the first place.

In the chapter on thermoregulatory adaptations, the classical description of Bergmann's rule is presented and interpreted as an adaptation to heat loss. Alternative explanations developed in recent years by evolutionary ecologists are not mentioned. Although thermogenesis is discussed, the concept of acclimatization is not presented in the context of adaptations to temperature. Unfortunately, acclimation and acclimatization are narrowly discussed in the context of physiological response to altitude.

Although the importance of scaling is presented in several contexts, it is not fully developed and its adaptive significance remains obscure and is sometimes misleading. The regression relating oxygen consumption to body size is presented with $b=0.75$. But the figure to which the reader is referred is a semilog plot of weightspecific oxygen consumption that produces a markedly different curve than that for which the slope was given. Finally, there is no discussion of the adaptive significance of the body size:metabolism relationship or of its relationship to life history theory.

The text succeeds much better as an introductory text. In general I found the writing clear, uncluttered, and geared for the student with no previous physiology. The introductory chapter succinctly outlines the development of physiology as a discipline. Unfortunately, we are left with names, dates, and contributions but with no feeling for the experimental procedure or observational techniques of these pioneers. The illustrations generally are well chosen and clear and there is a quantitative pervasiveness throughout the text. The 17-page glossary is helpful but has some unexpected omissions (e.g., ectothermy, but not endothermy, is defined). The attempt to keep matters simple sometimes is misleading (e.g., hibernation includes in its definition the statement "reduction in body temperature and metabolism of some animals that are temperature regulators when active," which incorrectly implies that temperature is not regulated during hibernation.

The selected bibliography consists almost entirely of review articles or advanced texts. The student receives no introduction to the research literature or journals where mammalian physiology is published.

In conclusion, the book is a useful beginning text for those who wish to emphasize the mechanisms of organ system function of mammals with some comparative and environmental additions. But other texts provide a better environmental and adaptive framework.

KENNETH B. ARMITAGE
Division of Biological Sciences
University of Kansas
Lawrence, KS 66045

\section{NEURAL DEVELOPMENT}

Molecular Bases of Neural Development. G. M. Edelman, W. E. Gall, and W. M. Cowan, eds. John Wiley \& Sons, New York, 1985. 606 pp., illus. $\$ 85.00$ (cloth).

The central question posed by the classical embryologist remains much the same: By what means does nature use the meeting of two individual cells to generate the stunning diversity and complexity of a mature organism? For the developmental neurobiologist the basic question takes a somewhat 